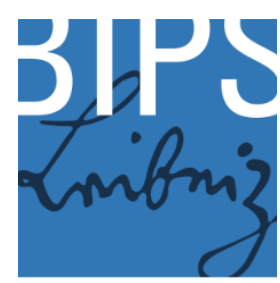

Leibniz Institute

for Prevention Research and

Epidemiology-BIPS

\title{
Overlooking informal dementia caregivers' burden
}

Oliver Riedel, Jens Klotsche, Hans-Ulrich Wittchen

\section{DOI}

10.10.3928/19404921-20160531-02

\section{Published in}

Research in Gerontological Nursing

\section{Document version}

Accepted manuscript

This is the author's final accepted version. There may be differences between this version and the published version. You are advised to consult the publisher's version if you wish to cite from it.

\section{Online publication date}

13 June 2016

\section{Corresponding author}

Oliver Riedel

\section{Citation}

Riedel O, Klotsche J, Wittchen H-U. Overlooking informal dementia caregivers' burden. Res Gerontol Nurs. 2016;9(4):167-74. 


\section{Dementia: Overlooking the informal caregiver's burden?}

Oliver Riedel, $\mathrm{PhD}^{1,2}$, Jens Klotsche, $\mathrm{PhD}^{3}$, Hans-Ulrich Wittchen, $\mathrm{PhD}^{2}$

${ }^{1}$ Leibniz Institute for Prevention Research and Epidemiology - BIPS, Bremen, Germany

${ }^{2}$ Technische Universitaet Dresden, Institute of Clinical Psychology and Psychotherapy

${ }^{3}$ Deutsches Rheuma-Forschungszentrum, Leibniz-Institut, Berlin

\section{Corresponding author:}

PD Dr. Oliver Riedel, Dipl.-Psych.

Leibniz Institute for Prevention Research and Epidemiology - BIPS

Achterstrasse 30, 28359 Bremen, GERMANY

Phone: ++ 49 421/21856883

E-Mail: riedel@bips.uni-bremen.de

\section{Acknowledgments:}

This study was supported through an unrestricted educational grant by Novartis (Nuremberg, Germany). Our thanks go to all participating physicians, patients and their caregivers. We would also like to thank Angela Emmrich, Bibiana Neumann, and Gabriela Zander-Schneider for their help in collecting the data.

\section{Conflict of interest}

Oliver Riedel and Jens Klotsche declare that there are no conflicts of interest. Hans-Ulrich Wittchen has received an unrestricted educational grant by Novartis for the conduction of this study. The sponsor had no influence on the study design, collection, analysis or interpretation of data, writing the report or the decision to submit the report for publication. 


\section{Summary}

Patients with Alzheimer's dementia (AD) need early support by caregivers (CGs). While CGs often have poor health, they mostly do not have time to seek medical advice for their own conditions. The patients' physicians, who are frequently the sole medical practitioner the CGs contact regularly, have an important function in recognizing the burden in CGs. We investigated to what extent they recognize the CGs' problems. In a two-staged survey in neurology outpatient care, CGs of patients with mild or moderate AD were enrolled into the study and appraised by the physicians regarding their physical and mental burden as well as need for help and advice about AD. Subsequently, caregivers' mental health was evaluated in a comprehensive diagnostic interview by blinded psychologists of the study center. Overall, 71.1\% of CGs had at least one somatic condition, and $43.7 \%$ of CGs had clinically relevant depressive symptoms; of these, $37.5 \%$ met criteria for major depression. Physicians rated $55.5 \%$ and $33.9 \%$ of CGs as not being physically or mentally comorbid. Among CGs with major depression, 33\% were appraised to require no professional help. Our findings suggest that the burden of a substantial proportion of affected caregivers is overlooked.

Keywords: Alzheimer, dementia, caregiver burden, depression, mental disorders 


\section{Introduction}

Dementias are characterized by progressing deterioration of cortical and subcortical functions, leading to cognitive decline and severe disabilities. The most common form is Alzheimer's disease (AD), representing approximately $70 \%$ of all dementias (Berr et al. 2005). As with progressing disease, patients lose their abilities to master daily life, family members are an important cornerstone in patient assistance - at great physical, emotional, and financial costs - and in prolonging the time prior to necessary institutionalization (Colerick and George 1986; Stone et al. 1987). However, providing informal care to a patient with dementia is challenging and the adverse impact of caring on family caregivers (CGs) is well documented. Compared with non-CGs, CGs of patients with AD have poorer mental and physical health and increased mortality risk (Dunkin and Anderson-Hanley 1998; Hiel et al. 2015; Mausbach et al. 2007; Schulz and Beach 1999). This has been confirmed by a recently published longitudinal study (Hajek \& König in press), and a systematic review has underscored the strong association between caregiver burden and depression (Van der Lee et al. 2014). This negative impact might be even stronger considering the fact that CGs of patients with dementia are often older, experiencing their own age-related medical conditions. Concurrently, as the provision of care becomes increasingly time-consuming, opportunities for socializing decrease (Papastavrou et al. 2015). A lack of social relationships, however, has been reported to have an impact on mental health as well as mortality comparable to well-established risk factors (Holt-Lunstad et al. 2010; Piercy et al. 2013). Given this increased morbidity of CGs and the direct link between care continuation and the CG's own health and well-being (Thomas et al. 2004), it is essential to support family CGs in their work and to identify health-related problems and other incapacities that might impede care provision. At the same time, however, as a consequence of the care situation, CGs frequently do not have time to seek medical advice for their own conditions. Here, the demented patients' physicians have, in addition to the actual patient's treatment, an important function in recognizing the negative impacts of caregiving on those providing the care (Adelman et al. 2014; Cohen 2000; O'Connor 2011). While the physician's responsibility is naturally limited to health issues of the patient, he or she might still have a good - and in some cases perhaps the only - opportunity to recognize and address mental health issues in the CGs arising from the care situation. Yet, to what extent the CGs' needs and problems are recognized by physicians is unexplored. Previous work suggests variance between CGs' physicians' perceptions regarding efficacy in providing disease support and information to the CG (American Alzheimer's Association 2001; Yaffe et al. 2008). For example, while GPs state their self-image of supporting the families of patients with dementia 
(Bruce et al. 2002), evidence exists that family CGs complain about a lack of emotional support and inadequate education on managing the patient's AD medication (Fortinsky 2001). Conversely, physicians have also reported that family CGs are frequently unreceptive to their suggestions on seeking help or assistance.

While these studies are valuable for identifying potential communication problems between CGs and physicians, they are mostly based on small sample sizes and often restricted to qualitative approaches, comparing the CGs' and physicians' expressed attitudes and expectations on support provision in the caregiving situation (Bruce et al. 2002; Schoenmakers et al. 2009; Yaffe et al. 2008). So far, associations between physician assessments and CGs' self-rated difficulties arising from the caring situation have not been adequately explored. Keeping in mind that CGs potentially hesitate to seek timely adequate help (Bruce et al. 2002), statistically based evidence from studies on the scope of the problem are essential for raising awareness about CGs who might substantially benefit from proactively offered help in their situation.

\section{Methods}

\section{Study design}

The design of the IDEA study has been described elsewhere in greater detail (Riedel et al. 2012). Briefly, the study was conducted in two stages. At stage one, office-based neurologists included patients with AD (McKhann et al. 1984) in either mild or moderate stages and their accompanying CGs during their daily routine care. To optimally reflect the daily home care situation, exclusion criteria for the patients were as minimal as possible and restricted to: higher stages of $\mathrm{AD}$ than moderate, consultation due to an acute emergency (e.g., pain), or lack of consent (either by the patient or the legal representative/CG). CGs of every age were eligible if they provided regular informal care to the patient. After inclusion, the physician clinically described each patient on a patient documentation sheet. Each CG was provided a CG questionnaire and asked to complete it during the waiting time. It comprised basic information on the CG, patient, and crude questions about the current home care situation. Afterwards, the patient documentation sheet and the CG questionnaire were mailed back to the study center. At stage two of the study, CGs were then contacted and interviewed by independent blinded members of the study team (clinical psychologists). This comprehensive interview then enlarged on the CG's health status and detailed aspects of the caregiving situation. A more detailed description of information collected at the two stages is given in the next section. 
In stage one, $n=403$ patient-CG dyads were included and documented. In stage two, $n=237$ CGs participated in interviews. CGs who disagreed did so due to lack of time (23\%), worries about privacy (9.8\%), or other reasons (26.3\%; e.g., emotional strain, logistic problems), while $40.9 \%$ did not state a reason for non-participation. CGs who only participated in stage one of the study did not differ from those who completed stage two in sex distribution ( $\mathrm{p}=$ $.060)$, age $(\mathrm{p}=.694)$, or duration of caregiving $(\mathrm{p}=.104)$. In additional, the associated patients did not differ regarding sex distribution $(p=.978)$, age $(p=.118)$, or duration and severity of $\mathrm{AD}(\mathrm{p}=.959$ and $\mathrm{p}=.765$, respectively).

\section{Study material}

Patient-related and CG-related data were collected at both stages of the study. At stage one, data were obtained from the treating physician (patient documentation sheet) and the CG (questionnaire). At stage two, the data source was the CG alone. Here, CGs were comprehensively interviewed by trained psychologists, who were blinded against the physician's appraisal.

\section{Patient-related data}

The physician provided patient-related data by completing a patient documentation sheet, which comprised basic sociodemographic information (e.g., sex, age, educational level, acknowledged care level). Moreover, the physician coded in detail the current score on the Mini-Mental State Exam (MMSE) and the current AD severity (Folstein et al. 1975). The CG documented the patient's functional status in instrumental activities of daily living and according to the Barthel-Index (BI) scale in the CG questionnaire (Lawton and Brody 1969; Mahoney and Barthel 1965). Using ten items, the BI scale assesses the patient's capability to eat, move, and to groom oneself without assistance. Total possible scores can range from 0 to 100, with lower scores indicating increased disability, i.e., higher dependence on assistance by CGs. In the interview, CGs provided more detailed information on the caring situation (e.g., service use, dementia's interference with daily care routine, and further patient morbidities).

\section{$C G$-related data}

In addition to patient characterization, each physician was also asked to rate the CG's burden, physical and psychiatric morbidity, and need for assistance on eight items in total. Regarding 
burden, the physician appraised 1) emotional strain, 2) physical strain, and 3) time burden of the caregiving situation on the CG. Each item was rated on a four-point scale ("very," "moderately," "mildly," "not at all"). Items four and five appraised the CG's physical and mental comorbidity on a four-point scale ranging from "normal" to "severe." Three items recorded the CG's need for support from the physician's perspective, in particular: "To what extent does the CG seek help?"; "To what extent does the CG need advice?"; and "Does the CG need medical/psychological treatment?" Again, each item was rated from "very" to "not at all."

The CG's personal information, including age, marital status, and relationship with the patient, was documented by the CG on the CG questionnaire. Additionally, each CG appraised physical health and emotional state on a scale analogue to the EuroQoL-5D Visual analogue scale (VAS), ranging from 0 to 100 with “100” indicating best state (Greiner et al. 2003).

At stage two, the CG interview focused on the current home care situation and addressed the CG's own mental and physical health. For depression screening, the Depression Screening Questionnaire (DSQ) was implemented (Wittchen et al. 2001). In this paper, a DSQ-score $\geq$ 10 indicated the presence of a major depression episode. CGs having a DSQ-score $\geq 8$ and $<$ 10 were classified as having a subthreshold depressive disorder.

\section{Statistical analysis}

Continuous variables are reported by means and standard deviations and categorical variables by absolute and relative frequencies. Differences in means were tested by univariate analysis of variance or the Mann-Whitney Test as appropriate. The agreement between the physician and CGs regarding the CG's psychiatric comorbidity, time burden, need for advice, and somatic comorbidity was estimated by the weighted kappa $(\kappa)$ statistics for categorical ratings and intraclass correlations (ICCs) for continuously distributed ratings. The ICCs were estimated after fitting a random-intercept model with the inclusion of the rater identifier (physician or CG) as a level two variable. Statistical inference was based on a significance level of 5\%. All statistical analyses were conducted with SAS 9.3 (SAS Institute Inc., Cary, NC).

\section{Ethical approval}


The study was conducted in compliance with the Declaration of Helsinki and had ethical approval from the Ethics Committee of Medical Faculty of the Technische Universität Dresden (Vote No. EK 75032009, July 4, 2009). Written informed consent was obtained from all participants.

\section{Results}

\section{Characteristics of the CG-patient-dyads}

The CGs and patients enrolled are characterized in Table 1. In more than two-thirds of dyads, females provided care; almost half of CGs were spouses of the associated patients, while $36.3 \%$ were sons/daughters. The mean duration of care was 40.1 months. The majority of CGs lived with the patient and were significantly older than those CGs who did not (67.9 vs. 52.3 years, $\mathrm{p}<.001)$. Regarding somatic comorbidity, the vast majority of CGs $(73.7 \%)$ had at least one medical condition and $44.8 \%$ had two or more. The most common comorbidities reported by CGs were cardiovascular diseases (18.1\%), hypertension (16.8\%), arthrosis $(10.8 \%)$, gastrointestinal diseases $(8.2 \%)$, and herniated discs and other unspecific back pain (7.8\% each).

- Table 1 -

The associated AD patients were predominantly female (61.1\%) with a mean age of 77.6 years and a mean AD duration of 3.1 years. Two-thirds of patients had moderate AD; onethird were in the mild stage. The mean Barthel score was $73.1 \pm 25.5$.

\section{CG physical and psychological state}

\section{Comorbidity appraised by physician}

The CG comorbidity and need for assistance as appraised by the physician are shown in Table 2. The vast majority of CGs were rated to be at least moderately emotionally $(86.6 \%)$ and physically (61.6\%) strained by the care situation. In contrast, a minority of CGs were rated to be at least moderately physically or mentally comorbid (14.1\% and $28.1 \%$, respectively), and $55.5 \%$ and $33.9 \%$ were appraised not to be physically and mentally comorbid at all. 
- Table 2 -

\section{Comorbidity as appraised by the $C G$}

The CGs rated a mean value of $64.3 \pm 20.6$ (median: 70) for physical health and $59.3 \pm 22.9$ (median: 60) for emotional state, respectively, on the VAS. The mean DSQ score was $4.5 \pm$ 3.7. In total, $43.7 \%$ of all CGs had clinically relevant depressive symptoms (subthreshold depression: $27.3 \%$; major depression: 16.4\%). While caregiving spouses and children had similar rates of major depression ( $16.0 \%$ vs. $19.8 \%$ ), children significantly more often suffered from subthreshold depression (33.7\% vs. $23.1 \%$ ) resulting in significant higher total rates of depressive symptoms in caregiving children than in spouses $(53.5 \%$ vs. $38.9 \%, \mathrm{p}<.05)$. Spouses with depressive symptoms were significantly younger than those without (68.5 vs. 72.9 years, $\mathrm{p}<.01$ ), while children CGs with and without depression did not differ in this regard (51.4 vs. 52.1 years, $\mathrm{p}=.690)$. No differences between depressed and non-depressed CGs were found for the duration of caregiving $(p=.292)$, somatic comorbidities $(p=.137)$, patient age $(p=.356)$, AD duration $(p=.745)$, the patient's Barthel score $(p=.318)$, or the patient's MMSE score $(\mathrm{p}=.516)$

\section{Need for information}

Regarding the degree of information on the nature of $\mathrm{AD}, 66.1 \%$ of CGs stated they were "well" or "very well" informed, 24.8\% felt "moderately" informed, and 9.1\% stated "worse" or "not at all" informed. Regarding the administration of AD medication, 74.9\% of all CGs stated that they were well informed about it, 18.1\% were "a little" informed, and 7\% "not at all."

Agreement between physician and $C G$

Among CGs with subthreshold depression and major depression, we investigated the proportion who were appraised by the physicians to be mentally comorbid (see Figure 1).

- Figure 1 - 
In both groups considered, $20 \%$ to $25.6 \%$ were evaluated to be not depressive at all, and $40 \%$ of CGs with major depression were evaluated to be mildly depressed only. Similarly, between $33.3 \%$ and $39.2 \%$ were appraised to be in no need of any medical/psychological treatment. Regarding the degree of information, among those CGs who felt less or not at all informed about the nature or the course of $\mathrm{AD}, 35 \%$ and $33 \%$ were appraised by the physician to have no or only mild need for information (data not shown).

We additionally analyzed the agreement between the physician and CGs regarding the CG's psychiatric comorbidity, time burden, need for advice, and somatic comorbidity by using kappa $(\kappa)$ statistics and ICCs. We found low rates of agreement for psychiatric comorbidity $(\kappa=$ $.36)$, appraisal of time burden $(\mathrm{ICC}=0.25)$, and need for advice $(\kappa=0.10)$ and a higher rate for the appraisal of the $\mathrm{CG}$ somatic comorbidity $(\mathrm{ICC}=0.81)$.

\section{Discussion}

We investigated the agreement between family CGs and physicians regarding appraisal of the CG's burden in the care situation. We found a high degree of physical as well as mental comorbidity among the CGs. Only one in four CGs did not suffer from a somatic condition. In fact, almost half of them suffered from two or more. Moreover, we found a high rate of depressive symptoms among the CGs, affecting more than $40 \%$ of them. More than one in three CGs even met the criteria for major depression according to DSM-IV, with higher rates for depression among caregiving children than among spouses. This dovetails with previous results from Conde-Sala and colleagues who reported higher burden and feelings of guilt in children than in spouses as well (Conde-Sala et al. 2010). Alternatively, a higher burden has also been found in caregiving spouses than in caregiving adult children (Pinquart and Sorensen 2003), suggesting that further factors probably mediate this association (e.g., the housing situation).

In addition to the increased morbidity of CGs, our data also suggest a substantial mismatch between the CG's conditions and the appraisals by the patient's physicians. From the physician's perspective, less than $30 \%$ were at least moderately mentally comorbid and even less than $15 \%$ were at least moderately physically comorbid. This corresponds with a potential underestimation of depression severity in CGs by the physicians. Among CGs with clinically relevant depressive symptoms, almost $30 \%$ were appraised to be not mentally comorbid and one-third of CGs were evaluated to be only mildly affected. These rates were comparable for 
CGs with major depression (20\% and $40 \%)$. Thus, between one quarter and one-third of CGs with serious mental disorders were missed by the physician and consequently were probably not recommended professional help for their condition or otherwise offered help. Our findings also show a potential lack of information in CGs regarding AD. At least one-third of all CGs who felt they were not well informed about the disease and its potential course were erroneously appraised by the physician as very well informed. However, recently published data obtained from surveys, qualitative studies and systematic reviews have just emphasized that knowledge on the nature and course of dementia is important to CGs (Boots et al. 2015; Jensen \& Inker 2015; Khanassov et al., 2016; Vaingankar et al. 2013). Also, the adverse impact of caregiving on the CG's mental health is substantially moderated by the CG's self-efficacy, which in turn largely depends on the individual's knowledge on the disease (Hajek \& König in press). Thus, our results stress the necessity of educating and counseling the CGs as timely as possible.

At first glance, our findings were not completely unexpected, as increased burden had earlier been reported in CGs of higher age and with mental and physical comorbidities (Dunkin and Anderson-Hanley 1998; Zanetti et al. 1998). In addition, previous work already suggested variance between CGs' and physicians' perceptions of efficacy in providing supporting and informing the CG on the disease (American Alzheimer's Association 2001; Schoenmakers et al. 2009; Yaffe and Klvana 2002). However, while these findings were mainly based on qualitative approaches and small samples, our study is the first to provide a more reliable quantification of the problem based on a larger sample of unselected CGs. Moreover, to the best of our knowledge, our study is also the first to investigate the recognition of comorbidities in CGs of patients with AD by the patient's physician.

When reviewing these results, it is also important to keep two aspects in mind. First, our enrollment of patient-CG dyads was restricted to patients with mild or moderate AD. By consequence, the comorbidity and burden of care is already substantial in early stages of the disease, when the patient still is comparably functional in terms of the Barthel scale. Second, it should be noted that, due to the high proportion of caregiving adult children, one-third of our CGs were still employed and therefore had to arrange the care activities with their jobs. The additional adverse impact of such a dual burden has been previously described and additionally increases the probability of an end to personal care (Fonareva and Oken 2014; Thomas et al. 2004). Thus, our results underline the necessity of early prevention measures also from a 
societal perspective, as they might help prolong the time until residential care becomes necessary as well as maintaining the CG's ability to work (Handels et al. 2013). Thus, an early detection of an unmanageable burden of care or even a full-blown depression is essential. There are several conceivable measures to do so. For example, both the CGs as well as the physicians should be made increasingly aware of the fact that CGs are prone to mental disorders and should actively seek or be offered medical advice as early as possible. Caregivers could be counseled by the patient's physician in this regard right from the beginning of their "caring career", i.e., immediately after the diagnosis of dementia. They could be regularly offered a brief depression screening when accompanying their relatives to their visits to the doctor. Also, other professionals who get in touch with demented patients and their family caregivers (e.g., mobile nursing services) could be educated about how to recognize early symptoms of depression and how to assist CGs in obtaining adequate help.

Some important limitations should be remembered when interpreting our results. Firstly, as already stated, our results are restricted to CGs of patients with mild or moderate AD. Patients with severe stages of the disease were not included and the situation of CGs providing care to such patients remains unknown. Secondly, we had a relatively high withdrawal rate from stage one to stage two of the study, mostly due to strain or without a reason stated by the CG. While our analyses did not detect differences between CGs and patients from both stages in core measures, depression screening was not available at stage one of the study. Thus, it cannot be excluded that the presence of depressive symptoms decreased the willingness to participate in the interview, leading to a potential underestimation in this respect in our results. Thirdly, due to its focus and methodological setting, the theoretical perspective of our study relied heavily on the influence of medical factors on caregiver burden. There is, however, substantial evidence that "non-medical" factors such as social relationships might contribute to the mortality risk of individuals to a degree comparable to well-established risk factors (Holt-Lunstadt et al. 2010). These aspects of social cognition can also be related to underlying neural and anatomical structures of the brain (Adolphs 2009). Thus, the lack of a measure of social cognition or social support in our study potentially limits the validity of our results. Finally, as the main limitation of our work, one should not lose sight of the fact that the physicians in our study were physicians of the patients and not of the CGs. Unlike discussed previously (Adelman et al. 2014), the physician's professional responsibility does not necessarily extend to the CG's health; it is highly probable that with thorough examination by a physician, a smaller proportion of morbid CGs would have been missed. However, despite these 
methodological and conceptual drawbacks, our results should be understood as emphasis on the valuable resource of the physician-CG interaction to mitigate $\mathrm{CG}$ burden. 


\section{References}

Adelman RD, Tmanova LL, Delgado D, Dion S \& Lachs MS. 2014. Caregiver Burden A Clinical Review. JAMA 311 : 1052-1059.

Adolphs R. 2014. The Social Brain: Neural Basis of Social Knowledge. Annu Rev Psychol 60 : 693-716.

American Alzheimer's Association. 2001. Alzheimers Disease Study - Communication gaps between primary care physicians and caregivers. Washington, DC: American Alzheimer's Association.

Berr C, Wancata J \& Ritchie K. 2005. Prevalence of dementia in the elderly in Europe. Eur Neuropsychopharmacol 15 : 463-471.

Boots LMM, Wolfs CAG, Verhey FRJ., Kempen G \& de Vugt. 2015. Qualitative study on needs and wishes of early-stage dementia caregivers: the paradox between needing and accepting help. Int Psychogeriatr, 27 : 927-936.

Bruce DG, Paley GA, Underwood PJ, Roberts D \& Steed D. 2002. Communication problems between dementia carers and general practitioners: effect on access to community support services. Med J Aust 177 : 186-188.

Cohen CA. 2000. Caregivers for people with dementia - What is the family physician's role? Can Fam Phys 46 : 376-380.

Colerick EJ \& George LK. 1986. Predictors of institutionalization among caregivers of patients with Alzheimers disease. J Am Geriatr Soc 37 : 493-498.

Conde-Sala JL, Garre-Olmo J, Turro-Garriga O, Vilalta-Franch J \& Lopez-Pousa S. 2010. Differential features of burden between spouse and adult-child caregivers of patients with Alzheimer's disease: An exploratory comparative design. Int J Nurs Stud 47 : 1262-1273.

Dunkin JJ \& Anderson-Hanley C. 1998. Dementia caregiver burden - A review of the literature and guidelines for assessment and intervention. Neurology 51 : S53-S60. 
Folstein M, Folstein S \& McHugh P. 1975. Mini-Mental state: a practical method for grading the mental state of patients by the clinician. J Psychiatr Res 12 : 189-198.

Fonareva I \& Oken BS. 2014. Physiological and functional consequences of caregiving for relatives with dementia. Int Psychogeriatr 26 : 725-747.

Fortinsky R. 2001. Health care triads and dementia care: integrative framework and future directions. Aging Ment Health 5 : S35-S48.

Greiner W, Weijnen T, Nieuvenhuizen M, Oppe S \& de Charro F. 2003. A European EQ-5D VAS valuation set. In The measurement and valuation of health status using EQ-5D - A European perspective, pp 103-142. Eds R Brooks, R Rabin \& F de Charro. Dordrecht: Kluwer.

Hajek A \& Konig HH. 2016. Informal Caregiving and Subjective Well-Being: Evidence of a Population-Based Longitudinal Study of Older Adults in Germany. JAMA, 17 : 300-305. doi: 10.1016/j.jamda.2015.10.015

Handels RLH, Wolfs CAG, Aalten P, Verhey FRJ \& Severens JL. 2013. Determinants of Care Costs of Patients With Dementia or Cognitive Impairment. Alzheimer Dis Assoc Dis 27 : 30-36.

Hiel L, Beenackers MA, Renders CM, Robroek SJW, Burdorf A \& Croezen S. 2015. Providing personal informal care to older European adults: Should we care about the caregivers' health? Prev Med 70 : 64-68.

Holt-Lunstad J, Smith TB \& Layton JB. 2010. Social Relationships and Mortality Risk: A Metaanalytic Review. PlosOne 7 : e1000316. Doi:10.1371/journal.pmed.1000316.

Jensen CJ, \& Inker J. 2015. Strengthening the Dementia Care Triad: Identifying Knowledge Gaps and Linking to Resources. Am J Alzheimers Dis Other Dement, 30 : 268-275.

Khanassov V \& Vedel I. 2016. Family Physician-Case Manager Collaboration and Needs of Patients With Dementia and Their Caregivers: A Systematic Mixed Studies Review. Ann Fam Med, 14 : 166-177. 
Lawton MP \& Brody EM. 1969. Assessment of older people: Self-maintaining and instrumental activities of daily living. Gerontologist 9 : 179-186.

Mahoney F \& Barthel D. 1965. Functional evaluation - The Barthel Index. MD State Med J14 : $61-65$.

Mausbach BT, Patterson TL, Rabinowitz YG \& Grant I. 2007. Depression and distress predict time to cardiovascular disease in dementia caregivers. Health Psychol 26 : 539-544.

McKhann G, Drachman D, Folstein M, Katzman R, Price D \& Stadlan EM. 1984. Clinical diagnosis of Alzheimer's disease - Report of the NINCDS-ADRDA work group under the auspices of department-of-health-and-human services task-force on Alzheimer's disease. Neurology 34 : 939-944.

O'Connor C. 2011. Caring for dementia carers: the role of general practitioners in Ireland. Irish J Med Sci 180 : 327-332.

Papastavrou E, Andreou P, Middleton N, Tsangari H, \& Papacostas S. 2015. Dementia caregiver burden association with community participation aspect of social capital. J Adv Nurs, $\mathbf{7 1}$ : 2898-2910.

Piercy KW, Fauth EB, Norton MC, Pfister R, Corcoran CD, Rabins PV \& Tschanz JT. 2013. Predictors of Dementia Caregiver Depressive Symptoms in a Population: The Cache County Dementia Progression Study. J Gerontol Ser B Psychol Sci Soc Sci, 68 : 921-926.

Pinquart M \& Sorensen S. 2003. Associations of stressors and uplifts of caregiving with caregiver burden and depressive mood: A meta-analysis. J Gerontol Ser B-Psychol Sci Soc Sci 58 : P112-P128.

Riedel O, Emmrich A, Klotsche J, Dodel R, Förstl H, Maier W, Reichmann H \& Wittchen HU. 2012. Alzheimer's Disease: Differences of transdermal versus oral treatment on caregiving time. Dement Geriatr Cogn Dis Extra 2 : 468-480. 
Schoenmakers B, Buntinx F \& Delepeleire J. 2009. What is the role of the general practitioner towards the family caregiver of a community-dwelling demented relative? Scand J Prim Health Care 27 : 31-40.

Schulz R \& Beach SR. 1999. Caregiving as a risk factor for mortality - The caregiver health effects study. JAMA 282 : 2215-2219.

Stone R, Cafferata GL \& Sangl J. 1987. Caregivers of the frail elderly - A national profile. Gerontologist 27 : 616-626.

Thomas P, Ingrand P, Lalloue F, Hazif-Thomas C, Billon R, Vieban F \& Clement JP. 2004. Reasons of informal caregivers for institutionalising dementia patients previously living at home: the Pixel study. Int J Geriatr Psychiatr 19 : 127-135.

Vaingankar JA, Subramaniam M, Picco L, Eng GK, Shafie S, Sambasivam R, Zhang YJ, Sagayadevan V \& Chong SA. 2013. Perceived unmet needs of informal caregivers of people with dementia in Singapore. Int Psychogeriatr 25 : 1605-1619.

van der Lee J, Bakker T, Duivenvoorden HJ \& Droes RM. 2014. Multivariate models of subjective caregiver burden in dementia: A systematic review. Ageing Res Rev, 15 :7693.

Wittchen HU, Höfler M \& Meister W. 2001. Prevalence and recognition of depressive syndromes in German primary care settings: poorly recognized and treated? Int Clin Psychopharmacol 16 : 121-135.

Yaffe MJ \& Klvana J. 2002. Physician perspectives on the elderly patient-family caregiverphysician encounter. Isr Med Assoc J 4 : 785-789.

Yaffe MJ, Orzeck P \& Barylak L. 2008. Family physicians' perspectives on care of dementia patients and family caregivers. Can Fam Phys 54 : 1008-1015.

Zanetti O, Frisoni G, Bianchetti A, Tamanza G, Cigoli V \& Trabucchi M. 1998. Depressive symptoms of Alzheimer caregivers are mainly due to personal rather than patient factors. Int J Geriatr Psychiatr 13 : 358-367. 


\section{Tables}

Table 1. Characteristics of caregivers (CG) and patients participating in the IDEA study.

\begin{tabular}{|c|c|}
\hline & Value \\
\hline \multicolumn{2}{|l|}{ Caregiver } \\
\hline Male:Female ratio $(\%)$ & $30.8: 69.2$ \\
\hline Age, years (mean \pm sd) & $62.1 \pm 13.6$ \\
\hline \multicolumn{2}{|l|}{ Degree of relationship to patient $(\%)$} \\
\hline Spouse & 48.5 \\
\hline Son / daughter & 36.3 \\
\hline Son-in-law / daughter-in-law & 5.5 \\
\hline Other & 9.4 \\
\hline Living with the Patient (\%) & 63.6 \\
\hline \multicolumn{2}{|l|}{ Occupational status (\%) } \\
\hline Retired & 48.7 \\
\hline Employed & 31.5 \\
\hline Jobless & 14.2 \\
\hline Early retired & 4.1 \\
\hline Unemployable & 1.5 \\
\hline Duration of Caregiving, months (mean $\pm \mathrm{SD}$ ) & $40.1 \pm 33.2$ \\
\hline \multicolumn{2}{|l|}{ Number of comorbid diseases $(\%)$} \\
\hline None & 26.3 \\
\hline One & 28.9 \\
\hline Two & 21.5 \\
\hline More than two & 23.3 \\
\hline \multicolumn{2}{|l|}{ Patient } \\
\hline Male:Female ratio (\%) & $38.9: 61.1$ \\
\hline Age, years (mean \pm sd) & $77.6 \pm 7.6$ \\
\hline Duration of $\mathrm{AD}$, years (mean $\pm \mathrm{SD})$ & $3.1 \pm 2.7$ \\
\hline \multicolumn{2}{|l|}{ Severity of AD (\%) } \\
\hline Mild & 34.4 \\
\hline Moderate & 65.6 \\
\hline Mini-Mental State Exam score (mean \pm SD) & $17.8 \pm 5.6$ \\
\hline Barthel Index $($ mean \pm SD) & $73.1 \pm 25.5$ \\
\hline $\mathrm{I}-\mathrm{ADL}$ score $($ mean $\pm \mathrm{SD})$ & $2.3 \pm 2.3$ \\
\hline Need of daily care by caregiver, minutes, Median (Q1; Q2) & $240(110 ; 600)$ \\
\hline
\end{tabular}


Table 2. Physicians' appraisals of the caregivers' (CG) burden, morbidity and need for assistance in the IDEA study.

Physicians appraisal of

\begin{tabular}{cccc}
\multicolumn{4}{c}{ Severity $(\%)$} \\
\hline Very/ & $\begin{array}{c}\text { Mode- } \\
\text { rate }\end{array}$ & Mild & $\begin{array}{c}\text { Not } \\
\text { at all }\end{array}$
\end{tabular}

\section{CG burden:}

How emotionally straining is the care situation?

37.9

48.7

$\begin{array}{ll}7.5 & 5.9\end{array}$

How physically straining is the care situation?

17.8

43.8

$17.3 \quad 21.1$

How time-consuming is the care situation?

37.9

44.7

$10.6 \quad 6.8$

\section{CG morbidity:}

Physical morbidity

Mental morbidity

$\begin{array}{llll}3.0 & 11.1 & 30.4 & 55.5 \\ 5.2 & 22.9 & 38.0 & 33.9\end{array}$

\section{CG need for support:}

Extent of seeking help

$\begin{array}{llll}11.2 & 33.1 & 23.2 & 32.5 \\ 25.2 & 39.0 & 17.1 & 18.7 \\ 11.4 & 22.4 & 21.3 & 44.9\end{array}$

Extent of needing advice

11.4

22.4

$21.3 \quad 44.9$

treatment 


\section{Figures}

Figure 1: Physicians' ratings of depression severity in caregivers with a subthreshold depressive disorder (DSQ score 8 or 9) or a major depression (DSQ score $\geq 10$ ). 


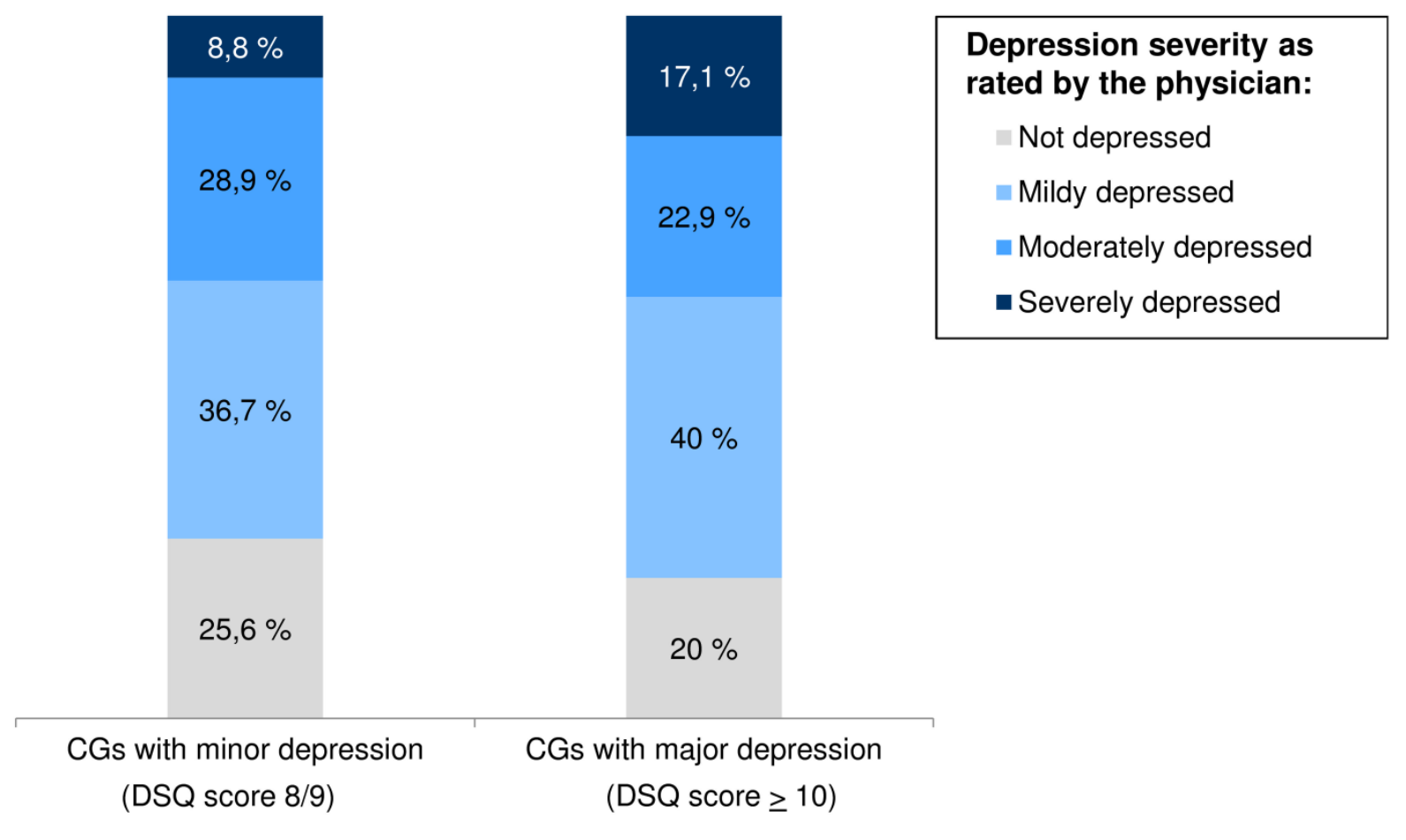

